\title{
Perfiles psicosociales de usuarios de entornos virtuales: motivaciones, conductas y consecuencias*
}

\section{Psychosocial Profiles of Users in Virtual Environments: Motivations, Behaviors, and Consequences}

Recepción: 13 Julio 2018 | Aceptación: 26 Junio 2019

\author{
Miguel Ángel Gandarillas Solinís ${ }^{\mathrm{a}}$ \\ Universidad Complutense de Madrid, España \\ ORCID: http://orcid.org/0000-0003-0943-4879 \\ Manuel Montañés Serrano \\ Universidad de Valladolid, España \\ ORCID: http://orcid.org/0000-0002-3107-8818
}

a Autor de correspondencia. Correo electrónico: mgandari@ucm.es

Para citar este artículo: Gandarillas-Solinís M. Á. \& Montañés-Serrano, M. (2019). Perfiles psicosociales de usuarios de entornos virtuales: motivaciones, conductas y consecuencias. Universitas Psychologica, 18(3), 1-14. https://doi.org/10.11144/Javeriana.ups y18-3.ppue

\section{RESUMEN}

Esta investigación tiene como objetivo identificar los tipos de usuarios habituales de los entornos virtuales, de acuerdo a las dimensiones motivacionales, características conductuales y sus consecuencias. Para tal fin se administró un cuestionario a 332 usuarios de plataformas virtuales. Los datos se procesaron con técnicas de análisis factorial, ANOVAs, conglomerados y regresiones múltiples, donde se identificaron cinco tipos de motivaciones del uso de las plataformas virtuales: la estimulación intelectual, el poder, instrumental, el anonimato/libertad, el afecto, y la evasión. Atendiendo a estos factores surgen cinco tipos de usuarios: el lógico, el anónimo, el honesto, el interesado, el evasivo, y el poderoso. Todo ello sugiere la posibilidad de distinguir dos dimensiones principales que explican el comportamiento virtual: la más relevante caracterizada por la búsqueda positiva de estimulación intelectual, creatividad y conocimiento, y una segunda de carácter afectivo-emocional, más relacionada con factores de vulnerabilidad y riesgo.

Palabras clave

Comportamiento virtual; motivación psicosocial; consciencia virtual; perfil usuario online; comunicación online.

\begin{abstract}
This study aims at identifying the main profiles of habitual users in virtual environments based on motivational dimensions, behavioral characteristics, and consequences. Data were gathered through a questionnaire from 332 users of online platforms and processed using factorial analysis, ANOVAs, cluster analyses and multiple regressions. Results suggest five types of motivations in online interaction: Intellectual stimulation, power, instrumental motives, anonymity/freedom, affection, and evasion. Five significant user-profiles groups around these factors, named: The Logical, the Anonymous, the Interested, the Evasive, and the Powerful. The results suggest two main dimensions explaining virtual behavior: One concerning the positive search for intellectual stimulation, creativity and knowledge and a second dimension more centered on
\end{abstract}


affective-emotional characteristics, more related to vulnerabilities and risks.

Keywords

Virtual Behavior; Psychosocial motivation; Virtual consciousness; Online user profile; Online communication.

Los primeros estudios sociales sobre el uso de las nuevas tecnologías de comunicación virtual se centraron en los impactos negativos para la persona, especialmente en los procesos de adicción y dependencia. Echeburúa, Amor y Cenea (1998) sostienen que la adicción a Internet comparte características propias de la dependencia física como son la pérdida de control, síndrome de abstinencia, interferencia en la vida cotidiana y pérdida de interés por otras actividades gratificantes. Situación que se agrava cuando la vulnerabilidad es mayor debida a problemas de personalidad, dificultad para las relaciones interpersonales o alteraciones psicopatológicas. Young (1998) distingue una serie de mecanismos psicológicos que pueden llevar a la adicción online como son la creación de una personalidad ficticia, el reconocimiento y poder, el apoyo social y la satisfacción sexual. Otros riesgos psicológicos muy estudiados están relacionados con el cyberbullying, el acoso online o el aislamiento social (Vivas \& Torres, 2011). Estudios psicosociales más recientes dan cuenta también de aspectos positivos relacionados con el uso de los entornos virtuales, como son el desarrollo de la consciencia (Gackenbach, 2006), la expresión, control interpersonal, el juego, el desarrollo de la identidad (Johnson \& Kulpa, 2007; Linares, Subrahmanyam, Cheng, \& Guan, 2011; Schimitt, Dayanim, \& Matthias, 2008), la socialización, la comprensión intercultural (Reich, Subrahmanyam, \& Guadalupe, 2012; Tuzel \& Hobbs, 2017; Willoughby, 2008), habilidades específicas como las de atención (Subrahmanyam, Greenfield, Kraut, \& Gross, 2001), el desarrollo intelectual, la motivación para aprender, el empoderamiento personal, la inteligencia colectiva (Guan \& Subrahmanyam, 2009; Tran \& Subrahmanyam, 2015; Willoughby, 2008; Woolley, Aggarwal, \& Malone, 2015), la resiliencia ante problemas asociados con las discapacidades (Suriá, 2015), y el uso terapéutico en personas con trastornos anímicos (Manzoni et al., 2016). Diferentes autores recogen los hallazgos de la investigación empírica en teorías parciales para explicar, por ejemplo, el comportamiento dependiente en medios digitales (Vivas \& Torres, 2011) o la preferencia de la comunicación "on-line" sobre la presencial (Johnson \& Kulpa, 2007).

Las investigaciones muestran cómo el comportamiento virtual ( $\mathrm{CV}$, aquel que se expresa en contextos no presenciales) aporta diferentes beneficios y riesgos, pero no aclaran suficientemente en qué casos se producen los beneficios y a quiénes puede afectar más negativamente. Esto es fundamental en cualquier estudio actual que supere la concepción clásica de comunicación social como mero intercambio de información, sin tener en cuenta las características de las personas intervinientes (Montañés, 2009). Es necesario avanzar en modelos más integrales que contemplen las características diferenciales de los usuarios y de sus usos, para así mejorar las cualidades de los medios virtuales, potenciando sus beneficios y reduciendo sus riesgos. Esta investigación tiene como objetivo definir la tipología de perfiles de usuarios de los entornos virtual de acuerdo con sus motivaciones, sus características de comportamiento en el medio virtual, y sus consecuencias.

La investigación en este campo se centra fundamentalmente en el estudio de dimensiones psicosociales. Esto se debe a que mientras otras prácticas y acciones (como el comportamiento del sueño, el comportamiento vial del riesgo, el comportamiento alimentico y digestivo o la defecación, etc.) son eminente individuales, el comportamiento virtual se encuentra condicionado, en buena parte, por las relaciones que se establecen entre el individuo y el grupo. Es por ello por lo que buena parte de los resultados de investigación sobre el CV encuentran fundamentos en la rica tradición de la psicología de los grupos (Festinger, 1981; Janis, 1987, Tajfel \& Turner, 1986). Los estudios relacionados con el CV identifican una serie de factores psicosociales que explican las diferencias 
en los comportamientos de los usuarios en los entornos virtuales (incluyendo aquí aplicaciones telemáticas, medios y soportes en redes sociales, juegos online, plataformas virtuales...), como son los siguientes:

1. Identidad y poder social. Algunos estudios señalan que las relaciones virtuales inciden diferencialmente en los usuarios en aspectos como la sociabilidad, la identidad social (Barker, 2009; Schimitt et al., 2008), y la búsqueda de sentimientos de poder grupal (Caplan, Williams, \& Yee, 2009), en ocasiones mediante la agresión, el "cyberbullying" o la exclusión virtual (Álvarez-García, Barreiro-Collazo, \& Núñez, 2017; Romera, Cano, \& García-Fernandez, 2016). El anonimato proporciona en ciertos usuarios la sensación de poder en el entorno virtual, inversamente relacionado con la cantidad de relaciones sociales y la autoestima en el entorno presencial (Keipi, Oksanen, \& Räsänen, 2014).

2. Consciencia y realidad virtual. La "realidad virtual" da sentido a la experiencia virtual ajustada a las características del entorno en el que ese comportamiento se manifiesta (Sherman \& Craig, 2002). Entendiéndose por consciencia virtual el conocimiento sobre la realidad virtual y sus limitaciones o "irrealidades", es decir, la capacidad de darse cuenta de esa realidad como propia de ese entorno virtual y no del entorno físico o presencial. Asociados a estos conceptos se encuentran los de inmersión (sensación de estar dentro de una realidad presencial cuando se utiliza un medio virtual) (Sherman \& Craig, 2002; Witmer \& Singer, 1998). Por el contrario, la sensación de sentirse en el medio virtual cuando uno se encuentra en un entorno presencial se entiende como disociación (Aardema, O'Connor, Côté, \& Taillon, 2010). Ésta es más probable cuando hay más implicación, control y atención selectiva por parte de la persona, y una mayor fidelidad en la imitación del entorno físico por parte del medio virtual (Witmer \& Singer, 1998). Estas cuestiones se relacionan con fenómenos de absorción psicológica (Murray, Fox, \& Pettifer, 2007).

3. Significado y transcendencia. La persona puede asignar a la experiencia virtual un mayor o menor significado $y$ transcendencia que afectará a su implicación e inmersión en el medio virtual, así como relevancia en su vida (Huang, Huang, Chou, \& Teng, 2017).

4. Interactividad. El ordenador y los móviles, como fuente de interacción interpersonal, pueden mejorar $\mathrm{O}$ empeorar las relaciones presenciales dependiendo de la persona (Rice, Sheperd, Dutton, \& Katz, 2007; VidalesBolaños \& Sádaba-Chalezquer, 2017).

5. Escape/evasión. Uno de los principales factores que pueden motivar el uso habitual de plataformas virtuales es el hecho de constituir un "mundo paralelo", una "vía de escape" de sus problemas (Pazukhina \& Filippova, 2014).

6. Compromiso presencial vs. virtual. El nivel de compromiso en el entorno virtual aparece altamente relacionado con los niveles de implicación emocional y "enganche" (Aristeidou, Scanlon, \& Sharples, 2017).

7. Vulnerabilidad vs. protección del yo. Diferentes estudios señalan una asociación entre tendencias hacia la ansiedad, depresión, exclusión social, o baja autoestima y el uso abusivo o agresivo de los dispositivos digitales (Álvarez-García et al., 2017; Jenaro et al., 2007), aunque la relación causaefecto entre estos factores y el CV no está clara (Morahan-Martin, 2005; Pawlak, 2002). El entorno virtual podría facilitar una mayor sensación de invulnerabilidad y protección de la autoestima (Ang, 2015). 
8. Reforzantes intelectuales y emocionales. Diferentes estudios han identificado elementos reforzantes psicosociales, como la retroalimentación positiva sobre la expresión y control de la propia identidad (Schimitt et al., 2008), o la mejora en la percepción de las relaciones sociales (Willoughby, 2008). Reforzadores positivos generales se relacionan con la interacción social, el desarrollo de la consciencia, el desarrollo intelectual, la creatividad, la inteligencia colectiva, o el apoyo emocional ante dificultades o problemas (Gackenbach, 2006; Serrano-Puche, 2016; Willoughby, 2008; Woolley et al., 2015). Por otro lado, las redes sociales online (social media) parecen reforzar y potenciar procesos grupales distorsionantes cuando ya están presentes inicialmente en el grupo, como son los de polarización y de pensamiento grupal (groupthink), tal y como están siendo estudiados en los fenómenos de transmisión de bulos/noticias falsas (fake news) o de desinformación (Garrett, Weeks, \& Neo, 2016; Lelkes, 2016; Suhay, Bello-Pardo, \& Maurer, 2018; Tewksbury, \& Riles, 2015).

9. Características personales y sociales diferenciales. Las características de cada persona conllevan una experiencia virtual diferente, $y$, por tanto, influyen en su forma de actuar, percibir e interpretar su realidad virtual. Beranuy, Carbonell, y Griffiths (2013) encontraron que los hombres eran más proclives al uso adictivo de los juegos en Internet, y las mujeres a usar más el móvil como medio para comunicar las emociones. También encontraron que ambas tecnologías eran más problemáticas en la adolescencia y se normalizaba con la edad, con menos consecuencias negativas. Los rasgos de personalidad como la extraversión, la abertura a la experiencia, la consciencia, la autoestima, o el narcisismo han sido también asociados a diferentes estilos de comunicación virtual (Marshall, Lefringhausen, \& Ferenczi, 2015).

10. Predisposiciones. Los estudios sobre predisposiciones se centran, por lo general, en aspectos negativos en los jóvenes. Así, por ejemplo, Ye y Lin (2015) encuentran una gran relación entre locus de control externo, soledad e infelicidad en el mayor uso de relaciones sociales virtuales.

11. Dependencias. El uso abusivo de la persona 'netdependiente' puede generar dependencia, aislamiento social, o distorsión de sus objetivos personales, familiares o profesionales (Young, 1998; Vivas \& Torres, 2011).

Esta investigación se centra en el análisis de las principales dimensiones psicosociales propias del CV. A tal fin se incluyeron los principales factores psicosociales identificados y se partió de la siguiente hipótesis: Los tipos de usuarios de los entornos virtuales se pueden distinguir de acuerdo con las diferentes motivaciones de uso, como son la búsqueda de relaciones afectivas, de información o conocimiento, de emociones, anonimato, afiliación y poder grupal, evasión o escape de la realidad o de la consciencia, y la búsqueda de invulnerabilidad. Los tipos de motivaciones y de usuarios predecirán significativamente las características conductuales, sus consecuencias y sus riesgos. En esta investigación, como se describe en el apartado siguiente dedicado a los métodos, los factores motivacionales son tratados como variables independientes y los indicadores de las características conductuales, sus consecuencias y sus riesgos como variables dependientes.

\section{Método}

\section{Diseño y procedimiento general}

Se definió un diseño y procedimiento de investigación estructurado en dos fases: primer estudio piloto preliminar y segundo estudio más amplio. 
El primer estudio preliminar piloto utilizó un cuestionario inicial (con ítems con escalas tipo Likert y escalas nominales) con indicadores de las dimensiones hipotetizadas, que sirvió para seleccionar los indicadores de mayor fiabilidad, validez, y representatividad, usando criterios estadísticos (análisis factorial) y conceptuales. La selección de la muestra se realizó en la plataforma Second Life (SL) ya que incluye de manera amplia las principales características del comportamiento virtual a estudiar y cuenta con un periodo largo de existencia, lo cual permitía la identificación de usuarios con diferentes tiempos de experiencia. Se seleccionaron aleatoriamente lugares en español y dentro de los lugares se entrevistaba a la primera persona que se encontrara en el lugar. Cuando la primera persona no accedía a ser entrevistada, se procedía a entrevistar a la siguiente, y así sucesivamente.

En el segundo estudio se administró un cuestionario más reducido (escalas tipo Likert y nominales) elaborado a partir del primer estudio, seleccionando los ítems según criterios estadísticos (análisis factorial) y conceptuales (revisión bibliográfica). La elección de los participantes se realizó de la misma manera que en el estudio preliminar. Se procuró recoger la información a diferentes horas del día y noche de forma distribuida.

\section{Muestra}

Se incluyeron 42 personas en el primer estudio piloto y 290 personas en el segundo estudio, todos ellos usuarios de plataformas virtuales. El tamaño de la muestra para el segundo estudio se estableció teniendo en cuenta el universo como infinito, se estimó un nivel de confianza de 2 $\sigma$, esto es, del $95.5 \%$, un margen de error de \pm $5.75 \%$, y una máxima dispersión, esto es, $\mathrm{p}=\mathrm{q}$.

\section{Variables}

Los indicadores del segundo estudio sobre los que se estudiaron los factores psicosociales fueron agrupados en cinco categorías, de acuerdo a la hipótesis formulada:
Descriptores sociodemográficos: Edad, estudios, sexo, estado civil, lugar de residencia.

Descriptores básicos del CV: Tipo/s de medio/ s virtual/es usado/s habitualmente, dedicación diaria, tiempo en años de uso del medio.

Motivadores del CV: Indicadores relacionados con los diferentes factores motivacionales hipotetizados y más relevantes en el estudio preliminar: creatividad, entretenimiento, sentimiento de poder, identidad y roles grupales, invulnerabilidad, información, anonimato, libertad, afecto, amistad, evasión, negocios, obtención de bienes, interés por el arte y cultura, práctica en idiomas.

Consecuencias del CV (el tipo de impacto que tiene en la persona dicho comportamiento): Cambio en las relaciones presenciales, cambio personal, identificación con el rol en el medio virtual, pertenencia grupal en el medio virtual, frecuencia de contacto social, trascendencia del medio virtual.

Indicadores de riesgo: Enganche, irrealidad virtual (sensación de que el medio virtual es más real que el presencial), asociación entre realidad virtual y presencial (nivel de disociación), preferencia por la comunicación virtual (cuando puede ser también presencial), y sentido de protección virtual.

\section{Procesamiento de los datos}

Los datos fueron introducidos en la base de datos de programa SPSS, realizándose los siguientes análisis:

Estadísticos descriptivos de todas las variables (frecuencias, medias, desviaciones típicas, histogramas).

Análisis factorial (método de extracción por análisis de componentes principales, rotación Varimax-Kaiser) de las variables motivacionales (con soluciones de 4 a 7 factores), seleccionando una solución de 6 factores según a criterios estadísticos y conceptuales.

Análisis de conglomerados (de $\mathrm{K}$ medias) con las puntuaciones factoriales de los factores motivacionales resultantes del análisis factorial seleccionado (con soluciones de 4 a 8 
conglomerados) para identificar una tipología de usuarios. Una solución de 6 factores fue seleccionado de acuerdo a criterios de conceptualización teórica, significatividad estadística de las diferencias entre grupos en cada factor (ANOVAS, cuyas pruebas $\mathrm{F}$ mostraron todas una $p<0.00$ ), y distribución igualitaria de casos entre grupos.

Regresiones lineales múltiples con los factores motivacionales como predictores de las variables del CV, consecuentes y de riesgo.

ANOVAS con los conglomerados (tipos de usuarios) como variables independientes y las variables del $\mathrm{CV}$, consecuentes, y de riesgo como variables dependientes.

\section{Resultados}

\section{Descriptores sociodemográficos de la muestra}

La edad media de los participantes fue de 31 años (desviación típica 9.5) — ver Figura 1 sobre la distribución de edades-, un 55\% hombres y un $45 \%$ mujeres; siendo un $68 \%$ solteros. El nivel educativo más frecuente es el de Secundaria (47\%).

\section{Figura 1}

Distribución de edades de la muestra en el segundo estudio.

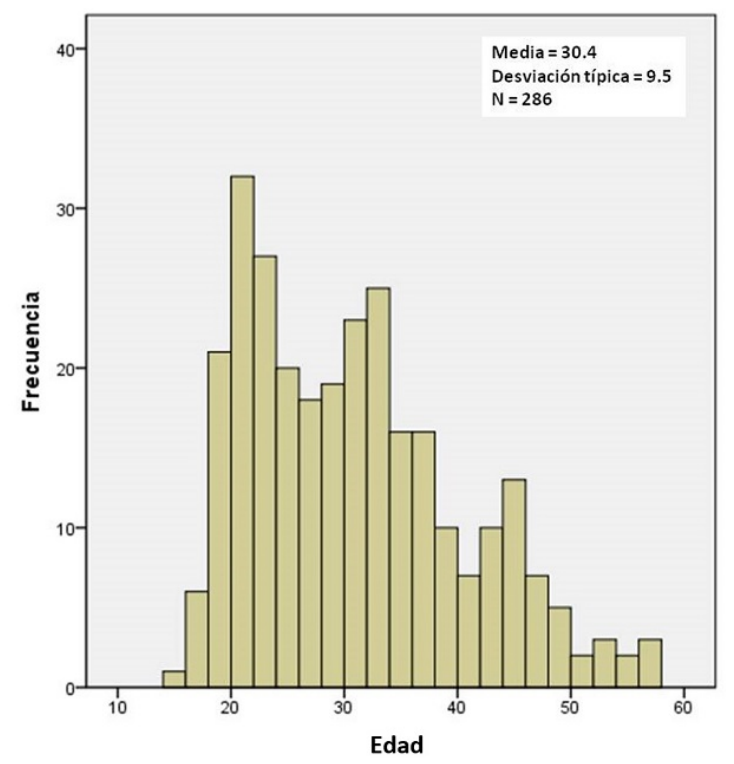

\section{Descriptores básicos del CV}

El 20\% de las personas se conecta más de cinco horas diarias. En cuanto a la evolución temporal en años de dedicación, aparece un incremento medio de uso hasta el primer año, y luego el tiempo dedicado disminuye. El 90\% de los usuarios no se relaciona presencialmente con ninguna persona de la plataforma virtual.

\section{Mediadores motivacionales}

Los factores que más se mencionan para elegir el uso de la plataforma virtual frente a la comunicación presencial fueron el entretenimiento, la amistad y el anonimato. Los factores más valorados en el uso de los medios virtuales son, en primer lugar, la amistad (3.0 sobre 5), en segundo lugar, la libertad (2.8) y, en tercer lugar, el entretenimiento (2.7). Entre los principales resultados, el $60 \%$ de los usuarios entrevistados se identifican bastante o mucho con su rol virtual. El 50\% expresó tener tanto relaciones sociales en el ámbito virtual como fuera de éste. Asimismo, se ha de decir que la mayoría de las variables muestran una gran distribución, lo que sugiere diferentes perfiles de usuarios.

En los análisis factoriales de los indicadores motivacionales se seleccionó la solución de 6 factores por coherencia y significación conceptual y teórica (ver Tabla 1), que reciben la siguiente denominación (en orden de importancia de los factores surgidos): 1) "Estimulación intelectual" (englobando variables relacionadas con la creatividad, artes y cultura, idiomas y entretenimiento); 2) "Poder" (incluye variables relacionadas con sentirse poderosos, posibilidad de representar papeles o personas y sentirse invulnerable); 3) "Razonamiento instrumental” (variables de negocios y la capacidad de poder conseguir cosas materiales); 4) "Anonimato" (libertad de expresión junto al anonimato que permiten los medios virtuales); 5) Afecto (relaciones afectivas y amistades); y 6) "Evasión" (incluyendo la variable de evasión). 
Tabla 1

Solución de 6 factores de las variables motivacionales

\begin{tabular}{|c|c|c|c|c|c|c|}
\hline \multirow[b]{2}{*}{$\begin{array}{l}\text { De la plataforma virtual } \\
\text { ¿Cuánto valoras... }\end{array}$} & \multicolumn{6}{|c|}{ Componentes } \\
\hline & 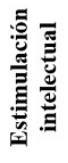 & : & 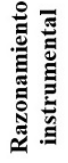 & 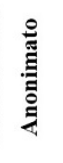 & 莺 & 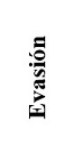 \\
\hline La creatividad & 0.78 & & & & & \\
\hline La cultura & 0.71 & & & & & \\
\hline Los idiomas & 0.67 & & & & & \\
\hline El entretenimiento & 0.61 & & & & & -0.51 \\
\hline Los sentimientos de poder & & 0.79 & & & & \\
\hline Representar papeles o personajes & & 0.68 & & & & \\
\hline La sensación de invulnerabilidad & & 0.67 & & 0.42 & & \\
\hline Los negocios & & & 0.81 & & & \\
\hline Poder conseguir cosas que no puedes & & 0.39 & 0.56 & & & \\
\hline La información & 0.39 & & 0.52 & & & \\
\hline El anonimato & & & & 0.85 & & \\
\hline La libertad & & & & 0.72 & & \\
\hline Las relaciones amorosas & & & & & 0.73 & \\
\hline La amistad & 0.3 & & & & 0.73 & \\
\hline Poder escaparte de los problemas & & & & & & 0.74 \\
\hline
\end{tabular}

Nota.Peso por debajo de 0.3 omitidos.

Tipos de usuarios

Los resultados del análisis de conglomerados (tipos de usuarios según factores motivacionales), de acuerdo con la solución seleccionada de 6 conglomerados, se muestran en la Tabla 2. Los tipos de usuarios se denominaron como sigue: 1) El Lógico (con mayores puntuaciones de los centroides de los factores motivacionales "Estimulación intelectual" y "Razonamiento instrumental"), 2) El Anónimo (con "Anonimato" como factor con más alta puntuación), 3) El Honesto (con "Anonimato" con más alta puntuación pero de signo negativo), 4) El Interesado (con "Estimulación Intelectual" con la puntuación más alta en signo negativo y "Razonamiento intelectual" como único factor en positivo), 5) El Evasivo (mostrando "Evasión" como factor con el centroide más alto), y 6) El Poderoso (con "Poder" como principal factor motivacional).
Tabla 2

Solución de 6 conglomerados de los factores motivacionales

\begin{tabular}{|c|c|c|c|c|c|c|}
\hline \multirow[b]{2}{*}{ Factores motivacionales } & \multicolumn{6}{|c|}{ Centros de conglomerados } \\
\hline & סֶ: & 竞 & 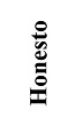 & 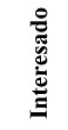 & 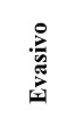 & 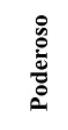 \\
\hline Estimulación intelectual & 1.01 & $-0,08$ & 0.47 & -0.97 & 0.41 & -0.64 \\
\hline Poder & 0.02 & 0.01 & -0.35 & -0.41 & -0.47 & 1.15 \\
\hline Razonamiento instrumental & 1.86 & -0.26 & -0.67 & 0.48 & -0.25 & -0.14 \\
\hline Anonimato & -0.03 & 0.91 & -1.07 & -0.93 & 0.52 & 0.07 \\
\hline Afecto & 0.31 & 0.01 & 0.34 & -0.74 & -0.79 & 0.66 \\
\hline Evasión & 0.18 & -0.8 & -0.24 & -0.3 & 1.22 & 0.77 \\
\hline $\mathbf{N}$ & 30 & 78 & 55 & 38 & 39 & 45 \\
\hline
\end{tabular}

Características de uso, consecuencias y riesgos

Entre los resultados descriptivos más significativos destacan los siguiente:

El 10\% piensa en las relaciones virtuales durante todo el día.

El $43.6 \%$ de los usuarios declara haber sido víctima de agresiones verbales.

El 23.9\% ha conocido personas que confunde la plataforma virtual con los medios presenciales.

El 35\% de las personas manifiesta que el sentido de trascendencia es igual que en su vida real.

El 50\% utiliza la plataforma virtual al menos alguna vez cuando lo puede hacer presencialmente.

El 25\% de los usuarios manifiesta que la plataforma virtual les protege más que la vida presencial.

\section{Predictores motivacionales}

En la Tabla 3 se ofrecen los resultados de las regresiones lineales múltiples con los factores motivacionales como predictores de los indicadores de $\mathrm{CV}$, consecuencias y riesgos. El nivel de protección percibida en el medio virtual se muestra como el indicador más significativo, en particular por los factores de poder $y$ anonimato, y en sentido contrario por el factor de evasión. El siguiente indicador en importancia es el tiempo de dedicación diaria, principalmente con el factor instrumental, aunque también con el intelectual, el afectivo y el de evasión como 
predictores significativos. Otros indicadores significativos son el aumento de relaciones presenciales y el de la asociación virtualpresencial. El mayor predictor motivacional es el factor afectivo, especialmente sobre el sentido de realidad virtual (comparado con la presencial), el sentido de pertenencia grupal virtual (comparado con el grupo presencial), y la asociación virtualpresencial. Con mayores niveles de afecto virtual aumentan las relaciones presenciales, según lo manifestado por los participantes. El factor Poder aparece como el segundo en importancia en su capacidad predictiva, especialmente sobre los indicadores de protección que ofrece el medio virtual, la trascendencia, y la frecuencia relativa (comparada con la presencial) de contacto social en el medio virtual. El factor instrumental sigue en importancia predictiva, especialmente respecto al número de horas de dedicación diaria, el grado de "enganche", la identificación con el rol virtual y la asociación virtualpresencial. Hay que reseñar que el factor de estimulación intelectual, aunque aparece como el más importante en el análisis factorial de motivadores, es uno de los que menos predice las consecuencias y riesgos.

\section{Tabla 3}

Regresiones múltiples. Resultados significativos de factores motivacionales prediciendo variables descriptoras de $\mathrm{CV}$, de consecuencias y de riesgo

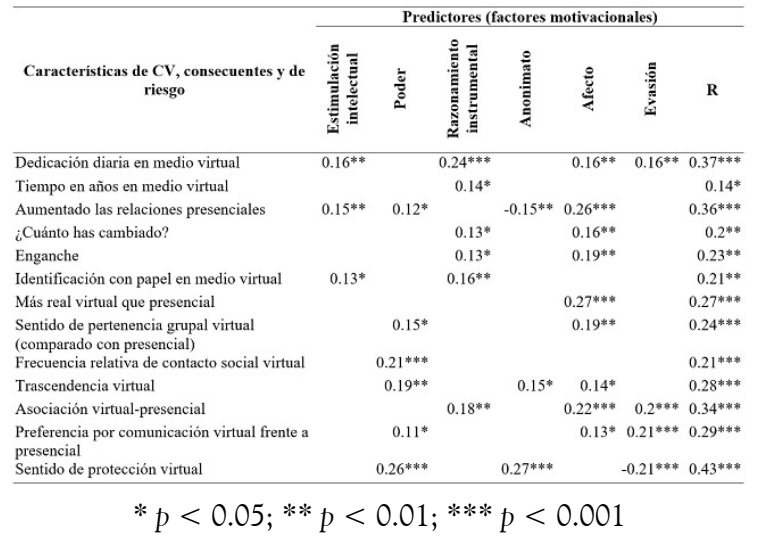

Diferencias entre tipos de usuarios

Hay diferencias significativas $(p<0.05)$ en las ANOVAS entre los tipos de usuarios (agrupados por los conglomerados) en las siguientes variables descriptoras del $\mathrm{CV}$, las consecuencias y los riesgos:

- Tiempo diario en la plataforma virtual (F (5, 27) $=4,41 ; p=0.001$ )

- Nivel de aumento o disminución de relaciones presenciales $(F(5.27)=3.23 ; p=$ 0.007)

- Nivel de identificación con rol virtual (F (5, 27) $=2.54 ; p=0.29$ )

- Sentido de realidad de la plataforma virtual $(F(5,27)=2.28 ; p=0.47)$

- Sentido de pertenencia grupal en el medio virtual $(F(5,27)=3.13 ; p=0.009)$

- Sentido de transcendencia en el medio virtual $(F(5,27)=3.79 ; p=0.002)$

- Asociación del medio virtual con el entorno presencial $(F(5,27)=6.92 ; p<0.000)$

- Preferencia por el medio virtual (frente a presencial) $(F(5,27) ; p=0.001)$

- Sentido de protección en el medio virtual $(F$ $(5,276)=5.58 ; p<0, .000)$.

La Figura 2 muestra las gráficas de estas diferencias significativas entre los tipos de usuarios sobre los indicadores que definen el uso, consecuencias y riesgos.

\section{Figura 2}

Diferencias significativas $(p>0.05)$ en las variables demográficas, variables descriptivas del comportamiento virtual, variables consecuentes y variables de riesgo entre los tipos de usuarios ("Lógico", "Anónimo", "Honesto", "Interesado", "Evasivo", y "Poderoso").

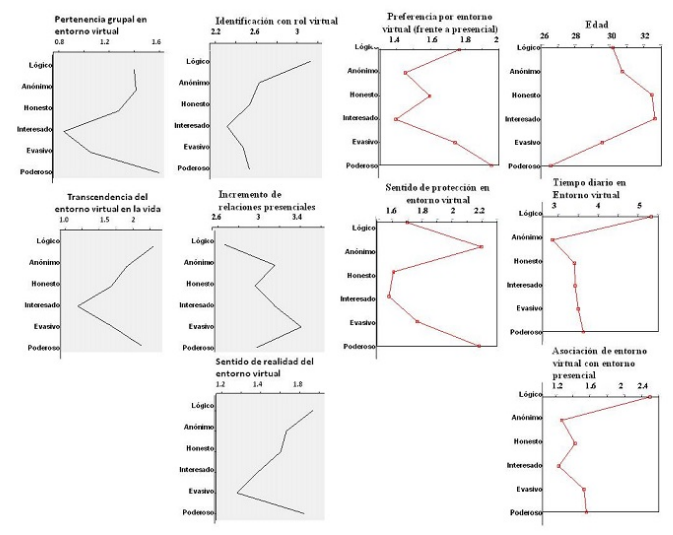

| Universitas Psychologica | V. I8 | No.3 | 2019 | 


\section{Discusión}

Los resultados apoyan parcialmente la hipótesis formulada. La diversidad de perfiles obliga a matizar las relaciones entre los factores psicosociales y reordenar la importancia del papel de cada uno. Los resultados apoyan, en parte, las hipótesis iniciales al sugerir la existencia de una principal dimensión motivadora, que tiene que ver con la búsqueda de estimulación intelectual, consciencia, creatividad, aprendizaje y conocimiento, que aparece como dimensión protectora y facilitadora del desarrollo psicosocial del usuario, en línea con los hallazgos de otros estudios mencionados en la introducción (Linares et al., 2011; Reich et al., 2012; Tran \& Subrahmanyam, 2015; Tuzel \& Hobbs, 2017; Willoughby, 2008; Woolley et al., 2015). Esta dimensión no parece tener grandes consecuencias en los elementos de riesgo, lo que sugiere principalmente cualidades positivas, con un efecto principal del CV en el desarrollo intelectual y cognitivo.

Los resultados también se muestran en la línea de las hipótesis iniciales al señalar una segunda dimensión general de implicación afectivoemocional que engloba factores afectivos $y$ factores de poder, anonimato, libertad, y evasión, estos últimos más relacionados con la protección y seguridad del entorno social virtual. Esta segunda dimensión tiene efectos tanto positivos como negativos, más asociada a fenómenos de riesgo que la primera dimensión, especialmente a aquellos relacionados con la "irrealidad" que tiende a mezclar los contextos presenciales con los virtuales, lo que ofrece un apoyo y explicación adicional a los estudios sobre el impacto diferencial de los medios online a nivel emocional (Álvarez-García et al., 2017; Aardema et al., 2010; Huang et al., 2017; Murray et al., 2007; Romera et al., 2016).

Los diferentes perfiles de usuarios que refleja este estudio también apoyan las hipótesis iniciales a mostrar la importancia de definir diferencialmente el CV teniendo en cuenta las características psicosociales de cada usuario. El polo intelectual frente al afectivoemocional marca de manera sobresaliente las diferencias entre grupos. En cambio, existe una relación curva entre grupos con respecto sus características de uso y consecuencias, con grupos con motivos más intelectuales (como el "Lógico") o emocionales (como el "Poderoso"), tendentes a mostrar similares puntuaciones, $\mathrm{y}$ grupos intermedios (como el "Interesado") con puntuaciones opuestas. Es posible que los grupos que expresan motivaciones más intelectuales y los de motivaciones más emocionales sean capaces de implicarse de forma similarmente intensa, aunque por motivos y con consecuencias diferentes.

Sobre resultados más específicos, cabe resaltar algunos hallazgos inquietantes, como que un $43.6 \%$ de usuarios expresa haber sido víctima de agresiones verbales, un $23.9 \%$ ha conocido personas que confunde la plataforma virtual con el entorno presencial, un $35 \%$ de las personas manifiesta que el sentido de trascendencia es igual que en su vida real o que el $25 \%$ de los usuarios manifiesta que la plataforma virtual les protege más que la vida presencial. Esto subraya la necesidad de analizar más en profundidad los riesgos y vulnerabilidades de diferentes formatos virtuales, sobre todo cuando se confunden entornos virtuales más artificiales y anónimos con los entornos presenciales, con una pérdida de la consciencia del entorno virtual, en concordancia con diferentes estudios (Aardema et al., 2010; Álvarez-García et al., 2017; Huang et al., 2017; Murray et al., 2007; Romera et al., 2016; Vivas \& Torres, 2011).

Los resultados también sugieren interesantes inferencias sobre qué perfiles de usuarios pueden ser más proclives y vulnerables al desarrollo de distorsiones cognitivas y/o emocionales. Vemos cómo los perfiles más emocionales, pero en particular el del "poderoso" transmite unas motivaciones relacionadas con unas conductas y unas consecuencias muy dirigidas a la necesidad de reconocimiento y estatus grupal. Esto podría explicar por qué hay personas y grupos que son más vulnerables a la influencia de las redes y entornos online en la generación de polarización y "pensamiento grupal" (group think), a acreditar "bulos/noticias falsas"" (fake news) y desinformación y a 
transmitirla (señalados por diferentes autores estudiando estos fenómenos, como Halberstam \& Knight, 2016; Lelkes, 2016; Shore, Baek, \& Dellarocas, 2016; Suhay et al., 2018) como procesos de búsqueda de pertenencia, identidad, poder y estatus grupal, ya ampliamente estudiados y teorizados por la psicología de los grupos de paradigmas como los de Festinger (1981), Janis (1987) y Tajfel y Turner (1986).

Para finalizar, en la Figura 3 se presenta el esquema de un modelo integral de comprensión de los procesos psicológicos en el $\mathrm{CV}$, con las principales dimensiones y su relación entre ellas, tal y como sugieren los resultados de este estudio. Se entiende el CV como un sistema procesual (con sus inputs, proceso, y outputs) que se retroalimenta desde mecanismos psicosociales tanto positivos (con factores más relacionados con la dimensión de estimulación intelectual, en verde, incluidos en la mitad superior del gráfico) como de riesgo (con factores más relacionados con la dimensión emocional, en morado, mitad inferior del gráfico), con los factores en azul y que pueden caer dentro de una dimensión u otra dependiendo de cada perfil del usuario. Así, el impacto de la comunicación virtual puede ser más positivo (desarrollo personal) o negativo (dificultades psicosociales) dependiendo del perfil psicosocial del usuario en el CV y de las características del entorno virtual utilizado.
Figura 3

Representación gráfica de modelo de relación entre dimensiones del CV en base a los resultados del estudio

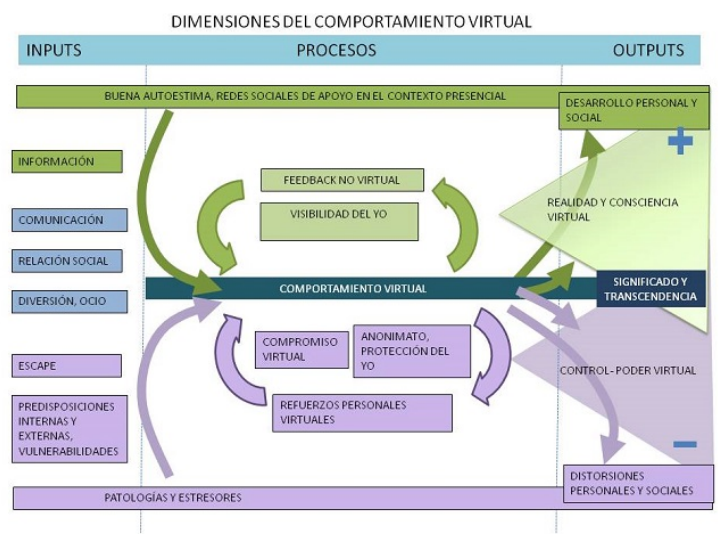

\section{Conclusiones}

Los resultados permiten concluir que los diferentes factores psicológicos que median en el CV pueden ser positivos siempre y cuando el usuario sea consciente de la realidad, cualidades, limitaciones y efectos (negativos y positivos) propios de cada medio virtual. El uso del medio será constructivo cuando el usuario sepa orientar su comportamiento hacia las cualidades positivas de cada medio, evitando ir más allá de lo que la realidad de ese medio puede ofrecer. En este sentido, la buena práctica y la mejora de la calidad de un medio debería facilitar el aprovechamiento de sus cualidades intrínsecas, sin pretender ir más allá de su propia realidad. De acuerdo con lo dicho, es fundamental para la calidad de un medio o soporte no pretender inducir cualidades o atributos de otros medios (virtuales o presenciales), enseñando así al usuario sus propias características, funciones de uso y limitaciones; permitiendo que cada tipo de usuario lo use de acuerdo a sus motivaciones; y promoviendo un uso adecuado y de prevención de usos "irreales" o distorsionantes del medio. 


\section{Limitaciones y Recomendaciones}

El estudio advierte sobre la importancia de orientar a los usuarios sobre las cualidades y riesgos de los diferentes medios virtuales, al saber identificar su perfil de CV. Al mismo tiempo, los resultados sugieren las grandes posibilidades que pueden tener un asesoramiento sobre cómo aprovechar los potenciales de estos medios para el desarrollo intelectual, emocional y social del usuario. Los medios virtuales deberían de incorporar mecanismos para facilitar sus cualidades y prevenir riesgos. Estos deberían de ser incorporados como indicadores de calidad del medio o plataforma.

Los resultados plantean la necesidad de superar el tipo de estudio todavía predominante, excesivamente reduccionista y negativista del uso de medios virtuales (centrados en los riesgos) a un estudio más amplio, centrado en el comportamiento. Así, proponemos que los estudios más clínicos se refieran a trastornos del comportamiento virtual, más que en la adicción o dependencia a los medios virtuales, y que también nos refiramos al desarrollo del comportamiento virtual, centrándonos en sus cualidades y potenciales psicosociales.

Una muestra mayor, incluyendo usuarios de otros medios virtuales (juegos on-line, chats, móviles...), sería necesaria para un mayor contraste de la validez de los resultados. Se considera necesario que los investigadores en el campo trabajen en la línea de diseñar herramientas de valoración de perfiles de CV para la orientación, asesoramiento, y counseling sobre los potenciales de desarrollo y sobre cómo prevenir riesgos de perfiles de usuarios determinados.

\section{Agradecimientos}

Los autores del artículo agradecen a Sonia Rojas, César Isasi, y Ana Casado su valiosa colaboración en la recogida de datos de este estudio.

\section{Referencias}

Aardema, F., O'Connor, K., Côté, S., \& Taillon, A. (2010). Virtual reality induces dissociation and lowers sense of presence in objective reality. Cyberpsychology, Behavior, and SocialNetworking, 13(4), 429-435. http s://doi.org/10.1089/cyber2009.0164

Álvarez-García, D., Barreiro-Colazo, A, \& Núñez, J.-C. (2017). Ciberagresión entre adolescentes: prevalencia y diferencias de género. Comunicar, 25(50), 89-97. https://d oi.org/10.3916/C50-2017-08

Ang, R. P. (2015). Adolescent cyberbullying: A review of characteristics, prevention andintervention strategies. Aggression and Violent Behavior, 25(A), 35-42. https://doi.o $\mathrm{rg} / 10.1016 / \mathrm{j} \cdot \mathrm{avb} .2015 .07 .011$

Aristeidou, M., Scanlon, E., \& Sharples, M. (2017). Profiles of engagement in online communities of citizen science participation. Computers in Human Behavior, 74, 246-256. https://doi.org/10.10 16/j.chb.2017.04.044

Barker, V. (2009). Older adolescents' motivations for social network site Use: The influence of gender, group identity, and collective self-esteem. CyberPsychology and Behavior, 12(2), 209-213. https://doi.org/10.1089/cp b.2008.0228

Beranuy, M., Carbonell, X., \& Griffiths, M. D. (2013). A qualitative analysis of online gaming addicts in treatment. International Journal of Mental Health and Addiction, 11 (2), 149-161. https://doi.org/10.1007/s11 469-012-9405-2

Caplan, S. E., Williams, D., \& Yee, N. (2009). Problematic Internet use and psychosocial well-being among $\mathrm{MMO}$ players. Computers in Human Behavior, 25, 1312-1319. https:/ /doi.org/10.1016/j.chb.2009.06.006.

Echeburúa, E., Amor, P.J., \& Cenea, R. (1998). Adicción a Internet: Una nueva adicción psicológica. Monografías de Psiquiatría, 2, 38-44.

Festinger, L. (1981). Human nature and human competence. Social Research: An International Quarterly, 48(2), 306-321. 
Gackenbach, J. I. (2006). Video game play and lucid dreams: Implications for the development of consciousness. Dreaming, 16(2), 96-110. https://doi.org/10.1037/105 3-0797.16.2.96

Garrett, R. K., Weeks, B. E., \& Neo, R. L. (2016). Driving a wedge between evidence and beliefs: How online ideological news exposure promotes political misperceptions. Journal of Computer-Mediated Communication, 21(5), 331-348. https://doi.org/10.1111/jcc4.1216 4

Guan, S. A., \& Subrahmanyam, K. (2009). Youth Internet use: Risks and opportunities. Current Opinions in Psychiatry, 22(4), 351-356. https://doi.org/10.1097/YCO.0b0 $13 \mathrm{e} 32832 \mathrm{bd} 7 \mathrm{e} 0$

Halberstam, Y., \& Knight, B. (2016). Homophily, group size, and the diffusion of political information in social networks: Evidence from Twitter. Journal of Public Economics, 143, 73-88. https://doi.org/10.1016/j.jpube co.2016.08.011

Huang, H. C., Huang, L. S., Chou, Y. J., \& Teng, C. I. (2017). Influence of temperament and character on line gamer loyalty: Perspectives from personality and flow theories. Computers in Human Behavior, 70, 398-406. https://doi.org/10.1016/j.chb.201 7.01 .009

Janis, I. L. (1987). Pensamiento grupal. Revista de Psicología Social, 2(1-2), 126-180. https://do i.org/10.1080/02134748.1987.10821566

Jenaro, C., Flores, N., Gómez-Vela, M., GonzálezGil, F., \& Caballo, C. (2007). Problematic internet and cell-phone use: Psychological, behavioral, and health correlates. Addiction Research Eु Theory, 15(3), 309-320.

Johnson, G. M., \& Kulpa, A. (2007). Dimensions of online behavior: Toward a user typology. CyberPsychology and Behavior, 10(6), 773-780. https://doi.org/10.1089/cp b.2007.0043

Keipi, T., Oksanen, A., \& Räsänen, P. (2014). Who prefers anonymous self-expression online? A survey-based study of Finns aged 15-30 years. Information, Communication $\mathbb{E}$
Society 18(6), 717-732. https://doi.org/10.1 080/1369118X.2014.991342

Lelkes, Y. (2016). Mass polarization: Manifestations and measurements. Public Opinion Quarterly, 80(S1), 392-410. https:/ /doi.org/10.1093/poq/nfw005

Linares, K., Subrahmanyam, K., Cheng, R., \& Guan, A.S. (2011). A Second Life within Second Life: Are virtual world users creating new selves and new lives? International Journal of CyberBehavior, Psychology and Learning 1(3), 50-71. https:/ /doi.org/10.4018/ijcbpl.2011070104

Manzoni, G. M., Cesa, G. L., Bacchetta, M., Castelnuovo, G., Conti, S., Gaggioli, A.... Riva, G., (2016). Virtual reality-enhanced cognitive-behavioral therapy for morbid obesity: A randomized controlled study with 1 year follow-up. Cyberpsychology, Behavior and Social Networks, 19(2),134-40. https://doi.org/10.1089/cyber.2015.0208

Marshall, T. C., Lefringhausen, K., \& Ferenczi, N. (2015). The Big Five, self-esteem, and narcissism as predictors of the topics people write about in Facebook status updates. Personality and Individual Differences, 85, 35-40. https://doi.org/10.1016/j.paid.2015. 04.039

Montañés, M. (2009). La comunicación desde la perspectiva sociopráxica. Mediaciones Sociales, (4), 51-74. Recuperado de https://revistas.ucm.es/index.php/MES O/article/view/MESO0909120051A

Morahan-Martin, J. (2005). Internet Abuse: Addiction? Disorder? Symptom? Alternative Explanations? Social Science Computer Review, 23(1), 39-48. https://doi. org/10.1177/0894439304271533

Murray, C. D., Fox, J., \& Pettifer, S. (2007). Absorption, dissociation, locus of control and presence in virtual reality. Computers in Human Behavior, 23(3), 1347-1354. https:/ /doi.org/10.1016/j.chb.2004.12.010

Pawlak, C. (2002). Correlates of Internet use and addiction in adolescents. Dissertation Abstracts International Section A: Humanities EJ Social Sciences, 63(5-A), 17-27. 
Pazukhina, S. V., \& Filippova, S. A. (2014). Problem of comprehensive study of psychological risks to children on the Internet. European Journal of Natural History, (2), 42-44. Recuperado de https://w orld-science.ru/en/article/view?id =33277

Reich, S.M., Subrahmanyam, K., \& Guadalupe, E. (2012). Friending, IMing, and Hanging Out Face-to-Face: Overlap in Adolescents' Online and Offline Social Networks. Developmental Psychology, 48(2), 356-368. h ttps://doi.org/10.1037/a0026980.

Rice, R. E., Sheperd, A., Dutton, W. H., \& Katz, J. E. (2007). Social interaction and the Internet: A comparative analysis of surveys in the US and Britain. In A. N. Joinson, K.Y.A. McKenna, T. Postmes \& U-D. Reips (Eds.), Oxford handbook of Internet psychology (pp. 7-30). Osford: Oxford University Press. https://doi.org/10. 1093/oxfordhb/9780199561803.013.0002

Romera, E. M., Cano, J. J., GarcíaFernández, C. M., \& Ortega-Ruiz, R. (2016). Cyberbullying: competencia social, motivación y relaciones entre iguales. Comunicar, 24(48), 71-79. https://doi.org/1 0.3916/C48-2016-07

Schimitt, K, Dayanim, S, \& Matthias, S. (2008). Personal homepage construction as an expression of social development. Developmental Psychology, 44(2), 496-506. h ttps://doi.org/10.1037/0012-1649.44.2.496

Serrano-Puche, J. (2016). Internet and emotions: New trends in an emerging field of research. Comunicar, 24(46), 19-26. https://doi.org/1 $0.3916 / C 46-2016-02$

Sherman, W. R., \& Craig, A. B. (2002). Understanding virtual reality: Interface, application, and design. San Francisco, CA: Morgan Kaufmann Publishers. Recuperado de https://www.elsevier.com/books/underst anding-virtual-reality/sherman/978-0-12-8 00965-9

Shore, J., Baek, J., \& Dellarocas, C. (2016). Network structure and patterns of information diversity on Twitter. Boston University Questrom School of Business
Research Paper No. 2813342, 1-67. http://d x.doi.org/10.2139/ssrn.2813342

Subrahmanyam, K., Greenfield, P., Kraut, R., \& Gross, E. (2001). The impact of computer use on children's and adolescent's development. Journal of Applied Developmental Psychology, 22(1), 7-30. https://doi.org/10.1016/S0193-3973( 00)00063-0

Suhay, E., Bello-Pardo, E., \& Maurer, B. (2018). The polarizing effects of online partisan criticism: Evidence from two experiments. The International Journal of Press/Politics, 23(1), 95-115. https://doi.org/10.1177/194 0161217740697

Suriá, R. (2015). Redes sociales online y perfiles resilientes en estudiantes universitarios con discapacidad. Anuario de Psicología, 45 (3), 317-330. Recuperado de https://www.raco.cat/index.php/Anuari oPsicologia/article/view/303462

Tajfel, H. \& Turner, J. C. (1986). The social identity theory of intergroup behavior. Psychology of intergroup relations, 5, 7-24.

Tewksbury, D., \& Riles, J. M. (2015). Polarization as a function of citizen predispositions and exposure to news on the Internet. Journal of Broadcasting $\mathbb{E}$ Electronic Media, 59(3), 381-398. https://doi.org/10.1080/08838151 .2015 .1054996

Tran, P., \& Subrahmanyam, K. (2015). Evidence-based guidelines for the informal use computers by children to promote the development of academic, cognitive and social skills. Ergonomics, 56(9), 1349-1362. https://doi.org/10.1080/001401 39.2013 .820843

Tuzel, S., \& Hobbs, R. (2017). The Use of Social Media and Popular Culture to Advance Cross-Cultural Understanding. Comunicar, 25(51), 63-72. https://doi.org/10.3916/C51 $-2017-06$

Vidales-Bolaños, M. J., \& Sádaba-Chalezquer, C. (2017). Adolescentes conectados: La medición del impacto del móvil en las relaciones sociales desde el capital social. Comunicar, 25(53), 19-28. https://doi.org/1 0.3916/C53-2017-02 
Vivas, G. P. M., \& Torres, F. C. (2011). Internet y pánico moral: revisión de la investigación sobre la interacción de niños y jóvenes con los nuevos medios. Universitas Psychologica, 10(3), 855. https://doi.org/10.11144/Javeri ana.upsy10-3.ipmr

Willoughby, T. (2008). A short-term longitudinal study of Internet and computer game use by adolescent boys and girls: Prevalence, frequency of use, and psychosocial predictors. Developmental Psychology, 44(1), 195-204. https://doi.org/10.1037/0012-164 9.44.1.195

Witmer, B. G., \& Singer, M. J. (1998). Measuring Presence in Virtual Environments: A Presence Questionnaire. Presence, 7(3), 225-240. https://doi.org/10.1162/10547469 8565686

Woolley, A. W., Aggarwal, I., \& Malone, T. W. (2015). Collective intelligence and group performance. Current Directions in Psychological Science, 24(6), 420-424. https: //doi.org/10.1177/0963721415599543

Ye, Y., \& Lin, L. (2015). Examining relations between locus of control, loneliness, subjective well-being, and preference for online social interaction. Psychological Reports, 116(1),164-175. https://doi.org/10. 2466/07.09.PR0.116k14w3

Young, K. S. (1998). Internet addiction: The emergence of a new clinical disorder. CyberPsychology Ė Behavior, 1(3), 237-244. https://doi.org/10.1089/cpb.1998.1.237

\section{Notas}

* Artículo de investigación. 